\title{
BMJ Open Protein misfolding, amyotrophic lateral sclerosis and guanabenz: protocol for a phase II RCT with futility design (ProMISe trial)
}

Eleonora Dalla Bella, ${ }^{1}$ Irene Tramacere, ${ }^{2}$ Giovanni Antonini, ${ }^{3}$ Giuseppe Borghero, ${ }^{4}$ Margherita Capasso, ${ }^{5}$ Claudia Caponnetto, ${ }^{6}$ Adriano Chiò, ${ }^{7}$ Massimo Corbo, ${ }^{8}$ Roberto Eleopra, ${ }^{9}$ Massimiliano Filosto, ${ }^{10}$ Fabio Giannini, ${ }^{11}$ Enrico Granieri, ${ }^{12}$ Vincenzo La Bella, ${ }^{13}$ Christian Lunetta, ${ }^{14}$ Jessica Mandrioli, ${ }^{15}$ Letizia Mazzini, ${ }^{16}$ Sonia Messina, ${ }^{17}$ Maria Rosaria Monsurrò, ${ }^{18}$ Gabriele Mora, ${ }^{19}$ Nilo Riva, ${ }^{20}$ Romana Rizzi, ${ }^{21}$ Gabriele Siciliano, ${ }^{22}$ Vincenzo Silani, ${ }^{23}$ Isabella Simone, ${ }^{24}$ Gianni Sorarù, ${ }^{25}$ Paolo Volanti, ${ }^{26}$ Giuseppe Lauria ${ }^{1}$

To cite: Bella ED, Tramacere I, Antonini G, et al. Protein misfolding, amyotrophic lateral sclerosis and guanabenz: protocol for a phase II RCT with futility design (ProMISe trial). BMJ Open 2017;7:e015434. doi:10.1136/ bmjopen-2016-015434

- Prepublication history for this paper is available online. To view these files, please visit the journal online (http://dx.doi org/10.1136/bmjopen-2016015434).

Received 6 January 2017 Revised 12 May 2017 Accepted 25 May 2017

CrossMark

For numbered affiliations see end of article.

Correspondence to Professor Giuseppe Lauria; giuseppe.lauriapinter@istitutobesta.it

\section{ABSTRACT}

Introduction Recent studies suggest that endoplasmic reticulum stress may play a critical role in the pathogenesis of amyotrophic lateral sclerosis (ALS) through an altered regulation of the proteostasis, the cellular pathwaybalancing protein synthesis and degradation. A key mechanism is thought to be the dephosphorylation of elF2 $\alpha$, a factor involved in the initiation of protein translation. Guanabenz is an alpha-2-adrenergic receptor agonist safely used in past to treat mild hypertension and is now an orphan drug. A pharmacological action recently discovered is its ability to modulate the synthesis of proteins by the activation of translational factors preventing misfolded protein accumulation and endoplasmic reticulum overload. Guanabenz proved to rescue motoneurons from misfolding protein stress both in in vitro and in vivo ALS models, making it a potential disease-modifying drug in patients. It is conceivable investigating whether its neuroprotective effects based on the inhibition of elF2 $\alpha$ dephosphorylation can change the progression of ALS. Methods and analyses Protocolised Management In Sepsis is a multicentre, randomised, double-blind, placebo-controlled phase II clinical trial with futility design. We will investigate clinical outcomes, safety, tolerability and biomarkers of neurodegeneration in patients with ALS treated with guanabenz or riluzole alone for 6 months. The primary aim is to test if guanabenz can reduce the proportion of patients progressed to a higher stage of disease at 6 months compared with their baseline stage as measured by the ALS Milano-Torino Staging (ALS-MITOS) system and to the placebo group. Secondary aims are safety, tolerability and change in at least one biomarker of neurodegeneration in the guanabenz arm compared with the placebo group. Findings will provide reliable data on the likelihood that guanabenz can slow the course of ALS in a phase III trial.

Ethics and dissemination The study protocol was approved by the Ethics Committee of IRCCS 'Carlo Besta Foundation' of Milan (Eudract no. 2014-005367-32 Preresults) based on the Helsinki declaration.

\section{Strengths and limitations of this study}

Amyotrophic lateral sclerosis (ALS) is a rare disease and randomised controlled trial (RCT) striving to obtain reliable data on the efficacy of candidate neuroprotective molecules should be performed with a multicentre design. Our consortium, including 25 ALS centres in Italy, satisfies the criteria of complementarily and synergy needed for a multicentre RCT. The coordinating centre is experienced in leading RCTs and the participating centres have a long-standing collaboration in the field of ALS, both in the clinical management of patients and clinical research. This will guarantee a standardised approach in all the patients.

- The study drug, guanabenz, has a mechanism of action close to pathogenic changes currently considered central to the pathogenesis of ALS, and preclinical studies are most promising.

- The futility design is an original and innovative methodological approach to neurodegenerative disease clinical trials. It allows optimising time and resources for a larger III phase study, but the a priori cut-off may be challenging.

- The primary outcome is a change in patients' function. It has been chosen to overcome the internal validity limitations of the ALS Functional Rating Score-Revised (ALSFRS-R) and to provide clinically meaningful results reflecting a concrete, though potential, advantage to patients. However, it may not be able to capture mild changes.

\section{INTRODUCTION}

\section{Background and rationale}

Recent evidence highlighted the crucial role that accumulation of misfolded protein aggregates in neurons and glial cells and failure of clearance mechanisms have in the 
pathogenesis of ALS. ${ }^{12}$ These aggregates have toxic properties and play a key role in the initiation and progression of cell loss. ${ }^{3}$ Protein components of these accumulations, found both in sporadic and familiar ALS, include TDP43, FUS/TLS and SOD1. ${ }^{4}$ The endoplasmic reticulum (ER) stress plays a critical role through the activation of the unfolded protein response (UPR) ${ }^{56}$ that regulates the proteostasis, ${ }^{7-9}$ namely the cellular balance between synthesis and degradation of proteins. The UPR is a cellular protective response against protein misfolding mediated by the upregulation of specific genes regulated by ER stress and the inhibition of protein translation. ${ }^{1011}$ However, long-term ER stress induces the activation of a parallel pathway that involves the activating transcription factor 4 (ATF4) and C/EBP homologous protein (CHOP) and leads to ER overload, with disruption of proteostasis and accumulation of misfolded proteins. Central to this mechanism is the increase of eukaryotic translation initiation factor 2A (eIF2 $\alpha$ ) phosphorylation. EIF2 $\alpha$ is a key factor for protein translation initiation ${ }^{12}$ and for the process known as integrated stress response (ISR). Altered eIF2 $\alpha$ phosphorylation, and therefore protein homeostasis, can affect the survival of cells. ${ }^{13}$ Among the different factors mediating this mechanism, the protein kinase RNA-like endoplasmic reticulum kinase (PERK) is considered a specific target, being one of the kinases that phosphorylates eIF2 $\alpha$ and coordinates ISR when the accumulation of misfolded proteins triggers the ER stress. ${ }^{14}$ Moreover, misfolded protein aggregates can activate a 'prion-like' mechanism of transmission between cells ${ }^{15} 16$ mediated by the activity of ribosomal RNA folding ${ }^{17} 18$ that recent data suggest to have a role in the progression of motor neuron death and therefore of ALS. ${ }^{19}$

Guanabenz, an alpha-2-adrenergic receptor agonist, blocks the dephosphorylation of eIF2 $\alpha$ and modulates the activity of ribosomal folding underlying aggregate transmission binding to the V-subunit ribosomal RNA (rRNA), thus reducing the accumulation of the aggregates and their transmission. ${ }^{18}$ The two effects of guanabenz as an alpha-2 agonist and modifier of protein misfolding are based on independent mechanisms of action. ${ }^{20}$ However, the pathway through which guanabenz exerts its effect of eIF2 $\alpha$ dephosphorylation eventually leading to the neuroprotection on motor neurons is unknown. It may be possible that guanabenz, through imidazoline receptors, activates a lysososmal degradation pathway ${ }^{21}$ and prevents motor neuron death. ${ }^{22}$

Therefore, it is conceivable to investigate whether its neuroprotective effects based on the inhibition of eIF2 $\alpha$ dephosphorylation and ribosomal modulation can change the progression of ALS.

Our phase II RCT will investigate, using a futility study design, clinical outcomes, safety, tolerability and biomarkers of disease progression in patients with ALS treated with guanabenz or riluzole alone for 6 months. Our primary aim is to obtain reliable data for a larger phase III trial. Guanabenz, formerly used as an antihypertensive agent, ${ }^{23}$ is an orphan drug (ORPHA178067) granted in 2009 by the European Medicines Agency for the treatment of traumatic spinal cord injury.

\section{Preliminary data}

Recent works suggested that ER stress-related activation of UPR leading to unbalance between protein production and degradation and spreading of toxic aggregates among motor neurons are two key mechanisms implicated in the pathogenesis of ALS. ${ }^{24}$ This hypothesis is supported both in sporadic and familiar ALS by the evidence of abnormal protein folding in motor neurons and sequestration of other proteins essential for cell functioning. Affected cells activate protective UPR response, which upstream components such as activated ER-resident transmembrane proteins (eg, PERK) attenuate global protein synthesis by phosphorylating eIF2 $\alpha$ and promote the translation of transcription factors (eg, ATF4, CHOP) which lead to the expression of PPP1R15A, an effector of negative feedback loop that terminates UPR signalling and dephosphorylates eIF2 $\alpha$ allowing protein synthesis to resume. UPR failure contributes to many pathological conditions that might be corrected by adequate boost of this adaptive response. Experimental data suggested that misfolding protein, RNA processing defects and toxic aggregation and spreading might converge on a common pathway leading to motor neuron degeneration. ${ }^{25-28}$ Most recently, salubrinal and guanabenz were found to counteract neuronal toxicity in worm and zebrafish models expressing mTDP-43 and mFUS through the reduction of ER stress response via UPR. ${ }^{29}$ These findings strengthened the hypothesis that ER stress pathway may be a crucial target for therapeutic interventions in ALS. In yeast, Drosophila and mouse models, guanabenz showed to modulate ribosome folding activity binding the V-subunit rRNA and reducing the prion-like propagation of aggregates. ${ }^{30}$

The therapeutic targets of guanabenz are the inhibition of stress-induced phosphatase and large subunit ribosome impairment. In this way, guanabenz can limit stress-induced protein synthesis and proteasome overload, allowing a correct cell clearance. This would also counteract mechanisms triggering intercell micropinocitosis, the seeds of spreading neurodegeneration. Salubrinal prevented muscle waste and prolonged survival in SOD1 mouse. ${ }^{26}$ However, salubrinal does not selectivity target ER-stress related phosphatase activity and is chemically unstable, making its clinical use unfeasible. Conversely, guanabenz selectively inhibits PPP1R15A and tunes translation in stressed cells, avoiding intolerable levels of eIF2 $\alpha$ phosphorylation and dismal low levels of protein synthesis. This rescue of proteostasis was found to protect motor neuron degeneration in in vitro and in vivo models along with the dramatically increased the levels of phosphorylated-eIF2 $\alpha$. Proteostatis balance is an important mechanism of cell survival. A large number of evidence suggests that ER stress-related protein misfolding is central to the pathogenesis of ALS. The first line of 
defence against the accumulation of misfolded proteins in the ER consists of phosphorylating the stress-induced eIF2 $\alpha$ phosphatase.

In the last 3 years, four papers on guanabenz and ALS have been published. The first showed that guanabenz enhanced the UPR and ameliorates mutant SOD1 ALS mice that had a delayed onset and prolonged survival. ${ }^{31}$ The second paper demonstrated that guanabenz delays the onset of disease symptoms, extends lifespan, improves motor performance and attenuates motor neuron loss in the SOD $1^{\mathrm{G} 93 \mathrm{~A}}$ mouse model of ALS, showing to attenuate ER stress and mitochondrial stress by prolonged eIF2 $\alpha$ phosphorylation. ${ }^{32} 33$ The third paper provided further evidence that protein phosphatase inhibition by guanabenz and Sephin 1 is effective in preventing in vitro motor neuron degeneration and in vivo disease progression. These data further strengthened the hypothesis that correcting proteostasis defects leading to accumulation of misfolded proteins could benefit a broad range of neurodegenerative diseases, including ALS. ${ }^{33}$

Finally, the fourth experimental paper reported a negative effect of guanabenz on ALS models. ${ }^{34}$ The authors showed that guanabenz is protective on fibroblasts expressing G93A mutant SOD1 when they are exposed to tunicamycin-mediated ER stress. However, in contrast to the previous reports, the authors reported that guanabenz accelerated disease progression in a mixed strain of mutant C57BL/6-SJL SOD1 transgenic mice. The authors reported altered behaviour in guanabenztreated mice of unknown significance, which might have been associated with the alternative route of administration by micropump infusion. The use of different strains, the possible different affinity for alpha2 and imidazoline receptors, the lack of data on motor neuron pathology and survival and on eIF2 $\alpha$ phosphorylation make the negative results arguable.

Preclinical data have demonstrated that proteostasis balance is an important mechanism of cell survival and ER-stress related protein misfolding is central to the pathogenesis of ALS. The first line of defence against the accumulation of misfolded proteins in the ER consists in phosphorylating the stress-induced eIF2 $\alpha$ phosphatase. In vitro data provided strong clues that guanabenz can spare the constitutive eIF2 $\alpha$ phosphatase and avoid persistent eIF2 $\alpha$ phosphorylation, which would be lethal to motor neurons, while it proved to ameliorate the course of the disease in two different ALS mouse models. Therefore, the bulk of in vitro and in vivo preclinical evidence in favour of a possible positive effect of guanabenz in patients with ALS is largely superior to the risk of a worsening of the disease.

\section{METHODS AND ANALYSIS}

Our phase II RCT will investigate, using a futility study design, clinical outcomes, safety, tolerability and biomarkers of disease progression in patients with ALS treated with guanabenz or riluzole alone for 6 months.
Our primary aim is to obtain reliable data for a confirmatory phase III trial.

\section{Objectives}

1. To investigate the effect of guanabenz as add-on treatment compared with riluzole alone in reducing the proportion of patients progressed to higher stages of disease over 6 months in sporadic or familial ALS patients;

2. To assess safety and tolerability related to alpha2 receptor agonist activity of guanabenz;

3. To explore serum biomarkers of ALS progression (creatinine, albumin) in guanabenz and riluzole alone arms at baseline and study end. In patients giving consent, the biomarkers will be explored also in the cerebrospinal fluid.

\section{Design of the study}

This paper is a multicentre, randomised, double-blind, placebo-controlled, phase II study with futility design. The trial has been designed following the guideline on clinical investigation of medicinal products for the treatment of ALS provided by the European Medicines Agency and adopted by the Agenzia Italiana del Farmaco (http://www.agenziafarmaco.gov.it/it/content/ linea-guida-sui-farmaci-iltrattamento-della-sclerosilaterale-amiotrofica-rilasciata-una-co). The protocol has been discussed and revised according to the suggestions of the Advisory Board. The Advisory Board of the study includes:

- Prof Orla Hardiman-Trinity College, University of Dublin

- Prof Paola Minghetti-University of Milan

- Dr Graziella Filippini-IRCCS Foundation 'Carlo Besta' Neurological Institute, Milan

- Dr Ettore Beghi-IRCCS 'Mario Negri' Institute, Milan.

The revised protocol has been presented to all the participating centres that have given their agreement during the Investigator Meeting held on 21 November 2014, at the Agenize Italiana per la Ricerca sulla Sclerosi Laterale Amiotrofica (AriSLA) headquarters, viale Ortles 21, Milan, Italy.

The study protocol was approved by the Ethics Committee of IRCCS Istituto di Ricovero e Cura a Carattere Scientifico on 28 October 2015 with Eudract Number 2014-005367-32. The authorisation of the Agenzia Italiana del Farmaco (AIFA) has been obtained on 1 March 2016 with protocol number AIFA/ $\mathrm{RSC} / \mathrm{P} / 20735$. Patient enrolment will start in December 2016.

The protocol has been design adhering to the Standard Protocol Items: Recommendations for Interventional Trials.

Figure 1 shows the flow-chart of the study

Patients diagnosed with probable laboratory supported or definite sporadic or familiar ALS according to the 


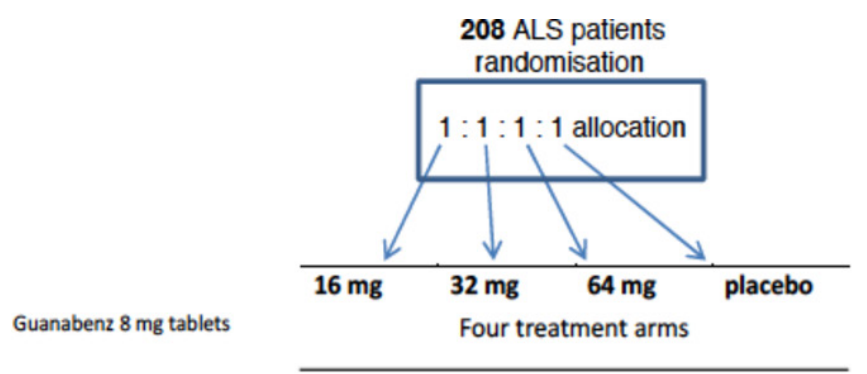

Figure 1 Flow-chart of the Protocolised Management In Sepsis study. ALT, Alanine transaminase; AST, Aspartate transaminase; BMI, body mass index; GGT, Gamma-glutamyl transpeptidase; SAE, serious adverse event; sVC, slow vital capacity.

revised El Escorial criteria ${ }^{35}$ will be assessed for eligibility (table 1).

\section{SAMPLE SIZE CALCULATION}

The sample size has been estimated as the proportion of patients progressing to higher stages of disease in 6 months as measured by the ALS Milano-Torino Staging (ALS-MITOS) system $^{36}$ in our cohort of 200 patients with ALS on riluzole randomised in the Erythropoietin in amyotrophic lateral sclerosis (EPOS): a multicentre, randomised, double blind, placebo controlled, phase III study and followed by the Consortium for 1 year. ${ }^{37} 38$ The ALS-MITOS is based on the loss of independent functions in the four key domains included in the ALSFRS-R (eg, walking/self-care, swallowing, communicating and breathing). This outcome can overtake the intrinsic limitations of the ALSFRS-R scale that is not unidimensional and has been proven not to meet the modern clinimetrics needs for a single scoring system in ALS. ${ }^{39}$ At baseline, 153 of 200 patients $(76.5 \%)$ were 
Table 1 Inclusion and exclusion criteria

\begin{tabular}{ll}
\hline Inclusion criteria & Exclusion criteria \\
\hline $\begin{array}{ll}\text { 1. Diagnosis of probable, probable laboratory supported or } \\
\text { definite sporadic or familiar ALS according to the revised EI }\end{array}$ & $\begin{array}{l}\text { 1. PEG, NIV or tracheotomy } \\
\text { Escorial criteria }\end{array}$ \\
$\begin{array}{ll}\text { 2. Age }>18 \text { years } & \text { 3. Known heart, renal or liver failure } \\
\text { 3. Onset } \leq 18 \text { months before randomization } & \text { 4. Known conditions at risk for cardiovascular disorders or } \\
\text { 4. sVC } \geq 70 \% \text { in spinal onset } & \text { symptomatic hypotension } \\
\text { 5. Riluzole } 100 \mathrm{mg} / \text { day or no riluzole } & \text { 5. Participation in a clinical trial within } 3 \text { months prior to the } \\
\text { 6. Written informed consent } & \text { screening }\end{array}$ \\
\hline
\end{tabular}

ALS, amyotrophic lateral sclerosis; NIV, non-invasive ventilation; PEG, percutaneous endoscopic gastrostomy; sVC, slow vital capacity.

in stage $0,44(22 \%)$ were in stage $1,3(1.5 \%)$ were in stage 2 and none was in stage 4 . At 6 months follow-up, $46.6 \%$ of patients progressed to a higher stage of disease. The ALS-MITOS system was found to predict at 6 months, irrespective of the number of functions lost, an event included in the primary outcome at 12 months with a probability of $82 \%$ and to correctly identify a patient who will not have an event included in the primary outcome with a probability of $63 \% .^{38}$

Based on these findings, we calculated the sample size. The null hypothesis is that guanabenz reduces the proportion of patients progressing to a higher stage of the disease at 6 months by at least $35 \%$ compared with their baseline stage and to the placebo group. The alternative hypothesis is that guanabenz reduces the proportion of patients progressed to a higher stages of the disease by less than $35 \%$ compared with their baseline stage and to the placebo group. If the null hypothesis were rejected, it would indicate that guanabenz is not sufficiently promising to change the progression of ALS in a phase III RCT, and in that sense is futile. The study was designed to reject the null hypothesis with an alpha of 0.1 and a power of 0.85 . For this purpose, a sample of 208 patients randomised in four treatment arms with allocation 1:1:1:1 (guanabenz $16 \mathrm{mg}$ + riluzole $100 \mathrm{mg}$; guanabenz $32 \mathrm{mg}$ + riluzole $100 \mathrm{mg}$; guanabenz $64 \mathrm{mg}$ + riluzole $100 \mathrm{mg}$; placebo + riluzole $100 \mathrm{mg}$ ) is needed.

\section{Randomisation}

The randomisation unit is at the coordinating centre. Eligible patients will be randomised in blocks and stratified by centre with 1:1:1:1 allocation in the four treatment arms. Patients will be allocated in one the following treatment arms:

1. guanabenz $16 \mathrm{mg}$ + riluzole $100 \mathrm{mg}$

2. guanabenz $32 \mathrm{mg}$ + riluzole $100 \mathrm{mg}$

3. guanabenz $64 \mathrm{mg}$ + riluzole $100 \mathrm{mg}$

4. placebo + riluzole $100 \mathrm{mg}$ daily.

The expected randomisation period will be of 12 months or until the achievement of the estimated number of patients. The randomisation unit will give the randomisation list exclusively to Cosmo Pharmaceuticals (Lainate, Milano, Italy; www.cosmopharma.com) responsible for the preparation of investigational drug and placebo.
The investigational drug/placebo will be dispensed to the pharmacy of each participating centre according to the randomisation allocation sequence. Treatment packs will be supplied for the entire study period along with information on how to administer the treatment. The randomisation unit will hold the treatment codes of each patients and will be available 24 hours a day over the entire study period to advise in an emergency whether a patient is receiving active or placebo.

\section{Experimental drug preparation}

The coordinating centre has purchased the active powder (guanabenz) by Medichem SA, Spain. Cosmo Pharmaceuticals (Lainate, Milano, Italy; www.cosmopharma. com) has prepared the active formulation at the different dosages (16 mg, 32 mg, 64 mg) and the placebo. The active powder and the investigational drug have been produced complying the Good Manufacturing Practices of the European Union for active pharmaceutical ingredients and ICH Q7A guidelines. Verum and placebo are identical and unrecognisable tablets by form size, weight and colour and will be indistinguishable to patients and neurologists. Tablets will be prepared in titration kits and boxes for the 6-month treatment.

\section{Treatment and blindness}

All patients will be treated for 6 months since the achievement of the dose at which they have been randomised (16 mg, $32 \mathrm{mg}$ or $64 \mathrm{mg}$ ). Active treatment will be started at the dose of $8 \mathrm{mg}$ daily and titrated up every 3 days to $16 \mathrm{mg}, 32 \mathrm{mg}$ or $64 \mathrm{mg}$ in 21 days. All patients will take the same number of tablets. Participating centres will receive the investigational drug packages for the entire study duration within 2 weeks after each patient randomisation. Treatment will be taken two times daily (morning and evening) for a period of 6 months. All patients will be required to take riluzole $100 \mathrm{mg}$ daily; patients will be eligible if at this stable dose for at least 30 days. Not to take riluzole for any reason will not be an exclusion criterion. Patients on riluzole at the randomisation will be asked to remain at the stable dose for the entire study period. Patients not on riluzole at randomisation will not be given riluzole for the entire study period. 


\section{PRIMARY OUTCOME}

Proportion of patients progressed to higher stages of disease at 6 months (measured by the ALS-MITOS system) by at least $35 \%$ in the guanabenz arm compared with their baseline stage and to the placebo arm (null hypothesis in the futility design).

\section{SECONDARY OUTCOMES}

1. Proportion of withdrawals due to adverse events $<20 \%$;

2. Decrease in mean decline of ALSFRS-R at 6 months in the guanabenz arms compared with placebo arm;

3. Difference in at least one serum biomarker of neurodegeneration (creatinine and/or albumin) comparing baseline and study end between guanabenz and placebo arm.

\section{COTREATMENTS}

Nutritional status and ventilation can affect survival and, therefore, the outcomes. We will strive to a homogeneous approach in all the participating centres.

\section{Nutrition}

The ultimate decision for a feeding tube placement will remain a very personal decision of each patient. However, malnutrition can affect survival. Therefore, percutaneous endoscopic gastrostomy (PEG) or equivalent device (eg, radiologically inserted device) will be discussed and proposed to all patients in the case of any of the following:

- Score 1 or 2 at item 3 of the ALSFRS-R;

- Unintentional loss of body weight $>10 \%$ in the last 3 months;

- Choking during ingestion of food, fluid or medication.

\section{Non-invasive ventilation}

Respiratory status declines in patients with ALS and non-invasive ventilation can improve survival. Symptoms suggestive of nocturnal hypoventilation (frequent arousals, morning headaches, excessive daytime sleepiness, vivid dreams) will be recorded in all patients. Non-invasive ventilation will be discussed and proposed to all patients in the case of any of the following:

- dyspnoea (score 0 or 1 at item 10 of the ALSFRS-R)

- orthopnoea (score 0 or 1 at item 11 of the ALSFRS-R)

- slow vital capacity $<50 \%$

- abnormal nocturnal oximetry $(\mathrm{SaO} 2<90 \%$ for $4 \%$ of the overnight recorded time).

\section{SAFETY ASSESSMENT}

Safety and tolerability of treatment will be controlled at every visit. Since the guanabenz is a hypotensive drug, blood pressure and side effect of overdose (severe dizziness, irritability nervousness, pinpoint pupils, low heartbeat, unusual tiredness or weakness) will be monitored $^{40}$ also through a questionnaire for side effects of guanabenz that will be given to each patient.

All patients will be provided a blood pressure diary to report measurements taken at home at least once a week and the information on when and how to measure the blood pressure and the interpretation of the results. Patients with symptomatic hypotension (eg, <90/60) interfering with daily activities and/or prelipotimic events and/or lipotimic events will be withdrawn. The Hamilton rating scale for depression ${ }^{41}$ will be performed in all patients at the enrolment visit and at monthly visit. Table 2 describes the safety assessment.

\section{Data recording and study monitoring}

All data will be recorded by an electronic case report form (eCRF). The study will be monitored by a certified contract research organisation (CRO).

\section{Statistical analysis}

Statistical analyses will be performed at the Neuroepidemiology Unit of the coordinating centre.

\begin{tabular}{|c|c|c|c|c|c|c|c|c|c|}
\hline & $\begin{array}{l}\text { Screening } \\
\text { visit }\end{array}$ & $\begin{array}{l}\text { Study-end } \\
\text { visit }\end{array}$ & $\begin{array}{l}\text { Drop-out } \\
\text { visit }\end{array}$ & Visit 1 & Visit 2 & Visit 3 & Visit 4 & Visit 5 & Visit 6 \\
\hline $\begin{array}{l}\text { Blood pressure } \\
\text { recording }\end{array}$ & $x$ & $x$ & $x$ & $x$ & $x$ & $x$ & $x$ & $x$ & $x$ \\
\hline ECG & $x$ & $x$ & $x$ & & & & & & \\
\hline $\begin{array}{l}\text { Haematological } \\
\text { exams (liver and } \\
\text { renal function) }\end{array}$ & $x$ & $x$ & $x$ & & $x$ & & $x$ & & $x$ \\
\hline Medical history & $\mathrm{x}$ & $x$ & $x$ & & & & & & \\
\hline $\begin{array}{l}\text { Signs and } \\
\text { symptoms of } \\
\text { overdose }\end{array}$ & & & & $\begin{array}{l}\text { X } \\
\text { (Weekly during } \\
\text { the titration } \\
\text { period- } 21 \text { day by } \\
\text { telephone) }\end{array}$ & $x$ & $x$ & $x$ & $x$ & $x$ \\
\hline Hamilton scale & $\mathrm{x}$ & & & $x$ & $x$ & $x$ & $x$ & $\mathrm{x}$ & $\mathrm{X}$ \\
\hline
\end{tabular}


Primary analysis of efficacy will be performed by intention to treat. Per-protocol analysis will be carried out after excluding non-compliers (patients who will take $<80 \%$ therapy). Statistics will be tabulated by treatment arm and time. Data will be analysed using t-test, Wilcoxon rank-sum test, $\chi^{2}$ test, Fisher's exact test or Kaplan-Meier estimator as appropriate. Statistical analyses will be carried out using SAS V.9.3 software (Stata Corporation). Randomisation and statistical analysis will be performed by different persons.

\section{Role of funding}

This is an academic, independent clinical research project funded by AriSLA. The pharma company from which guanabenz is purchased and the company that has prepared the investigation drug will not be involved at any level in the study. Participating units and patients will not be paid.

\section{ROLE OF PARTICIPATING CENTRES: PROMISE STUDY GROUP}

ALS is a rare disease and RCT striving to obtain reliable data on the efficacy of candidate neuroprotective molecules should be performed with a multicentre design. Our consortium includes 25 ALS centres in Italy and satisfies the criteria of complementarily and synergy needed for a RCT in ALS. The coordinating centre is experienced in leading RCTs and the participating centres have a long-standing collaboration in the field of ALS, both in the clinical management of patients and clinical research. It will guarantee a standardised approach to the diagnosis and management of the patients. All partners have participated in a recent phase III RCT led by the coordinating centre of the present RCT, and can guarantee efficient recruitment and low drop-out rates. Participating centres are not involved in other competitive trials. All centres are already provided of all the equipment needed to assess the patients during the RCT and have laboratory units available to store and ship the biological samples for biomarkers assays by means of consolidated protocols. The analysis of blood and CSF biomarkers will be centralised at the coordinating centre. Randomisation and statistics centres will be also centralised to orchestrate the different phases of the project. All trial activities will be monitored by a CRO.

Each centre is expected:

1. To randomise at least eight patients fulfilling including and excluding criteria in a period of 12 months and to administer the treatment for 6 months;

2. To provide one principal investigator and one neurologist to evaluate including and excluding criteria, administer treatment and assess primary and secondary outcome;

3. To formally adhere to the practice parameters of the American Academy of Neurology concerning the standardisation of the management of the patient in terms of ventilatory support and nutrition. Box reports the list of the participating centres.
Box List of the amyotrophic lateral sclerosis (ALS) centres, locoregional subinvestigators and Italian towns participating in the study. Thirteen Italian Regions will be represented and at least one ALS centre per regions will be involved, providing a large coverage of the national territory.

1. IRCCS 'Carlo Besta' Neurological Institute (Coordinating Centre)

2. ALS Centre, University of Torino

3. ALS Centre, University of Genova

4. ALS Centre, S. Raffaele Hospital, Milano

5. ALS Centre, 'S. Maugeri' Foundation, Milano

6. NEMO Clinical Centre, Milano

7. ALS Centre, IRCCS Istituto Auxologico Italiano, Milano

8. ALS Centre, University of Modena

9. ALS Centre, University of Siena

10. Neurology Unit, University of Ferrara

11. Neurology Unit, Hospital of Udine

12. NESMOS Department, Neuromuscular Disease Unit, Sant'Andrea Hospital and University of Rome 'Sapienza', Rome

13. ALS Centre, Cagliari

14. ALS Centre, 'S. Maugeri' Foundation, Mistretta (ME)

15. ALS Centre, second University of Napoli

16. ALS Centre, University of Padova

17. ALS Centre, University of Novara

18. Neurology Unit, Department of Neuro-Motor Diseases, IRCCS Arcispedale Santa Maria Nuova, Reggio Emilia

19. Department of Neurology, University of Pisa

20. ALS Centre, University of Palermo

21. Department of Neurology, University of Chieti

22. ALS Centre, Dpt of Neurology, University of Bari

23. NEMO SUD Clinical Centre, Messina

24. Casa di Cura Privata del Policlinico, ALS Centre, Milano

25. Neurology Unit, Spedali Civili, Brescia

\section{ADVERSE EVENTS}

All adverse events occurring between the first study-related procedure and the last study-related procedure will be reported. Those meeting the definition of serious adverse events (SAE) will be reported using the SAE Form. The coordinating centre will evaluate any safety information that is spontaneously reported by an investigator beyond the time frame specified in the protocol. All adverse events, regardless of seriousness, severity or presumed relationship to study therapy, will be recorded using medical terminology in the eCRF. Whenever possible, diagnoses should be given when signs and symptoms are due to a common aetiology (eg, cough, runny nose, sneezing, sore throat and head congestion should be reported as 'upper respiratory infection'). Investigators must record in the eCRF their opinion concerning the relationship of the adverse event to study therapy. All measures required for adverse event management must be recorded in the source document and reported according to sponsor instructions. The IRCCS Fondazione Istituto Neurologico 'Carlo Besta' assumes responsibility for appropriate reporting of adverse events to the regulatory authorities and reports to the investigators all SAE unlisted and associated with the use of the drug. The investigator (or coordinating centre where 
required) report these events to the appropriate Ethics Committee that approved the protocol unless otherwise required and documented by the Ethics Committee. In compliance with the Italian Health Ministry 'Decreto 17 dicembre 2004', the coordinating centre will provide in due time the pharmaceutical company entitled to sell the study product in Italy all information on observed serious adverse event, if any.

\section{ETHICS AND DISSEMINATION}

The study protocol was approved by the Ethics Committee of IRCCS 'Carlo Besta Foundation' and ALS-Consortium (Eudract no. 2014-005367-32) based on the Helsinki declaration. All subjects must sign and personally date an approved informed consent form after receiving detailed written and verbal information about the reason, the nature, the required procedures, the intended duration and the possible risks and benefits and any discomfort associated with the study. He/She should be informed that the subject's participation in the study is voluntary and that he/she may refuse to participate or withdraw from the trial, at any time, without penalty or loss of benefits to which the subject is otherwise entitled. The language used in the oral and written information about the trial, including the written informed consent form, should be as non-technical as practical and should be understandable to the subject or the subject's legally acceptable representative and the impartial witness, where applicable. The subject must be given ample time to read and to understand the patient information sheet and opportunity to inquire and ask any clarification about the trial before signing the informed consent form. Prior to a subject's participation in the trial, the written informed consent form should be signed and personally dated by the subject or by the subject's legally acceptable representative, and by the physician who conducted the informed consent discussion. When applicable, the investigator may conduct the informed consent discussion in presence of an impartial witness, who should sign and personally date the informed consent form. No study procedure can be performed before the written informed consent has been provided. The informed consent procedure must be done according to the guidelines provided in the Declaration of Helsinki and the ICH E6 Guideline for Good Clinical Practice.

The subject must be made aware and agree that personal information may be scrutinised during audit by competent authorities and properly authorised persons. However, personal information will be treated as strictly confidential and will not be publicly available. By signing the investigator statement the investigator assures the sponsor that informed consent will be obtained.

Any study subject may be withdrawn from the study at any time either at the discretion of the investigator or at the request of the study subject. However, the study subject is not under any obligation to provide a reason for withdrawal.
The trial has been designed following the guideline on clinical investigation of medicinal products for the treatment of ALS provided by the European Medicines Agency and adopted by the Agenzia Italiana del Farmaco (http:// www.ema.europa.eu/docs/en_GB/document_library/ Scientific_guideline/2013/07/WC500147005.pdf).

The protocol has been discussed and revised according to the suggestions of the Advisory Board

Results will be first presented and discussed with the participating centres, the scientific board of AriSLA and the Advisory Board. The results of the study will be presented at scientific symposia and published in scientific journals only after review and written approval by the involved parties in full respect of the privacy of the participating subjects. None of the investigators at the participating centres can make use of any information or data before, during and after the study without the written approval from the principal investigator.

\section{RELEVANCE TO PRACTICE/CONCLUSION}

Neuroprotective treatments able to slow and, hopefully, halt the progression of ALS are urgently needed. The objective of clinical researchers is to formulate RCTs on solid preclinical studies, with strong statistical design, feasible in terms of time and economic resources and with the ability to obtain results in the shortest time frame. It would improve our knowledge on the disease and generate hope in patients with ALS enhancing the therapeutic alliance and the service done to the community. We would like the Protocolised Management In Sepsis trial to be a promise in achieving these objectives.

\section{Author affiliations}

${ }^{1} 3$ rd Neurology Unit and ALS Centre, IRCCS 'Carlo Besta' Neurological Institute, Milan, Italy

${ }^{2}$ Scientific Direction, IRCCS 'Carlo Besta' Neurological Institute, Milan, Italy ${ }^{3}$ Neuromuscular Disease Unit, Sant'Andrea Hospital and University of Rome 'Sapienza', Rome, Italy

${ }^{4}$ Neurologic Unit, Monserrato University Hospital, Cagliari University, Cagliari, Italy ${ }^{5}$ Neurologic Clinic, SS Annunziata Hospital, Chieti, Italy

${ }^{6}$ Department of Neurosciences, Rehabilitation, Ophthalmology, Genetics, Mother and Child Disease, IRCCS University Hospital San Martino IST, Genova, Italy

${ }^{7}$ Department of Neurosciences, ALS Centre, 'Rita Levi Montalcini', University of Turin and Azienda Ospedaliero Universitaria Città della Salute e della Scienza, Turin, Italy

${ }^{8}$ Department of Neurorehabilitation, Casa Cura Policlinico, Milan, Italy

${ }^{9}$ Neurology Unit, S Maria della Misericordia University Hospital, Udine, Italy

${ }^{10}$ Neurologic Clinic, University of Brescia, Brescia, Italy

${ }^{11}$ Department of Medical and Surgery Sciences and Neurosciences, University of Siena, Siena, Italy

${ }^{12}$ Neurologic Clinic, University of Ferrara, Ferrara, Italy

${ }^{13}$ ALS Research Centre, BioNeC, University of Palermo, Palermo, Italy

${ }^{14} \mathrm{NEuroMuscular}$ Omnicenter, Serena Onlus Foundation, Milan, Italy

${ }^{15}$ Department of Neurosciences, S Agostino-Estense Hospital, Modena, Italy

${ }^{16}$ ALS Centre, Neurologic Clinic, Maggiore della Carità University Hospital, Novara;, Italy

${ }^{17}$ NEMO SUD Clinical Centre, Messina, Italy

${ }^{18} 2$ nd Neurologic Clinic, 2nd University of Naples, Naples, Italy

${ }^{19}$ ALS Center, 'Salvatore Maugeri' Clinical-Scientific Institutes, Milan, Italy

${ }^{20}$ Department of Neurology IRCCS 'San Raffaele' Hospital, Milan, Italy

${ }^{21}$ Neurology Unit, Department of Neuro-Motor Diseases, IRCCS Arcispedale Santa Maria Nuova, Reggio Emilia, Italy 
${ }^{22}$ Department of Clinical and Experimental Medicine, Neurology Unit, University of Pisa, Pisa, Italy

${ }^{23}$ Department of Neurology and Laboratory of Neuroscience, IRCCS Istituto Auxologico Italiano - Department of Pathophysiology and Transplantation, "Dino Ferrari" Center, Università degli Studi di Milano, Milan, Italy

${ }^{24}$ Department of Basic Medical Sciences, Neurosciences and Sense Organs, University of Bari, Bari, Italy

${ }^{25}$ Department of Neurosciences, University of Padua, Padua, Italy

${ }^{26}$ Intensive Neurorehabilitation Unit, IRCCS 'Salvatore Maugeri' Foundation, Mistretta, Italy

Contributors IT, EDB and GL: study concept and final design. All the coauthors of the PROMISE trial study group (namely ACò, CC, NR, GM, CL, VS, JM, FG, EG, RE, GA, $\mathrm{GB}, \mathrm{MF}, \mathrm{MC}, \mathrm{PV}$, MRMò, GSù, LM, RR, GS, VLB, MC, IS, SM) have contributed to the design of the protocol.

Funding The study is funded by the Agenzia Italiana per la Ricerca sulla Sclerosi Laterale Amiotrofica (AriSLA).

Competing interests None declared.

Patient consent Obtained.

Ethics approval AlFA/Advisory Board AriSLA/Ethics Committee of Promise study group.

Provenance and peer review Not commissioned; externally peer reviewed.

Open Access This is an Open Access article distributed in accordance with the Creative Commons Attribution Non Commercial (CC BY-NC 4.0) license, which permits others to distribute, remix, adapt, build upon this work non-commercially, and license their derivative works on different terms, provided the original work is properly cited and the use is non-commercial. See: http://creativecommons.org/ licenses/by-nc/4.0/

(c) Article author(s) (or their employer(s) unless otherwise stated in the text of the article) 2017. All rights reserved. No commercial use is permitted unless otherwise expressly granted.

\section{REFERENCES}

1. Ciechanover A, Kwon YT. Degradation of misfolded proteins in neurodegenerative diseases: therapeutic targets and strategies. Exp Mol Med 2015;47:e147.

2. Nordlund A, Oliveberg M. SOD1-associated ALS: a promising system for elucidating the origin of protein-misfolding disease. Hfsp J 2008;2:354-64

3. Ilieva H, Polymenidou M, Cleveland DW. Non-cell autonomous toxicity in neurodegenerative disorders: ALS and beyond. J Cell Biol 2009;187:761-72.

4. Polymenidou M, Cleveland DW. The seeds of neurodegeneration: prion-like spreading in ALS. Cell 2011;147:498-508.

5. Kanekura K, Suzuki H, Aiso S, et al. ER stress and unfolded protein response in amyotrophic lateral sclerosis. Mol Neurobiol 2009;39:81-9.

6. Walker AK, Atkin JD. Stress signaling from the endoplasmic reticulum: A central player in the pathogenesis of amyotrophic lateral sclerosis. IUBMB Life 2011;63:n/a-63.

7. Ruegsegger C, Saxena S. Proteostasis impairment in ALS. Brain Res 2016;1648(Pt B):571-9.

8. Maharjan N, Saxena S. ER strikes again: Proteostasis Dysfunction In ALS. EMBO J 2016;35:798-800.

9. Yerbury JJ, Ooi L, Dillin A, et al. Walking the tightrope: proteostasis and neurodegenerative disease. J Neurochem 2016;137:489-505.

10. Parakh S, Atkin JD. Protein folding alterations in amyotrophic lateral sclerosis. Brain Res 2016;1648(Pt B):633-49.

11. Wang L, Popko B, Roos RP. The unfolded protein response in familial amyotrophic lateral sclerosis. Hum Mol Genet 2011;20:1008-15.

12. Tsaytler P, Harding HP, Ron D, et al. Selective inhibition of a regulatory subunit of protein phosphatase 1 restores proteostasis. Science 2011;332:91-4.

13. Ron D, Harding HP. Protein-folding homeostasis in the endoplasmic reticulum and nutritional regulation. Cold Spring Harb Perspect Biol 2012; 4:a013177.

14. Ron D, Harding HP. elF2a phosphorylation in cellular stress responses and disease. In: Hershey JWB, Sonenberg N, Mathews $\mathrm{MB}$, eds. Translational control in Biology and Medicine. New York: Cold Spring Harbor Monograph Series: CSHL Press, 2007:345-68.
15. Verma A, Tandan R. RNA quality control and protein aggregates in amyotrophic lateral sclerosis: a review. Muscle Nerve 2013;47:330-8.

16. Verma A. Protein aggregates and regional disease spread in ALS is reminiscent of prion-like pathogenesis. Neurol India 2013;61:107-10.

17. Kanouchi T, Ohkubo T, Yokota T. Can regional spreading of amyotrophic lateral sclerosis motor symptoms be explained by prionlike propagation? J Neurol Neurosurg Psychiatry 2012;83:739-45.

18. Gitler AD, Shorter J. RNA-binding proteins with prion-like domains in ALS and FTLD-U. Prion 2011;5:179-87.

19. Tribouillard-Tanvier D, Béringue V, Desban N, et al. Antihypertensive drug guanabenz is active in vivo against both yeast and mammalian prions. PLoS One 2008;3:e1981.

20. Nguyen $\mathrm{PH}, \mathrm{Hammoud} \mathrm{H}$, Halliez S, et al. Structure-activity relationship study around guanabenz identifies two derivatives retaining antiprion activity but having lost $\alpha 2$-adrenergic receptor agonistic activity. ACS Chem Neurosci 2014;5:1075-82.

21. Choi SH, Choi DH, Lee JJ, et al. Imidazoline drugs stabilize lysosomes and inhibit oxidative cytotoxicity in astrocytes. Free Radic Biol Med 2002;32:394-405.

22. Casanovas A, Olmos G, Ribera J, et al. Induction of reactive astrocytosis and prevention of motoneuron cell death by the I(2)-imidazoline receptor ligand LSL 60101. Br J Pharmacol 2000;130:1767-76

23. Holmes B, Brogden RN, Heel RC, et al. Guanabenz. A review of its pharmacodynamic properties and therapeutic efficacy in hypertension. Drugs 1983;26:212-29.

24. Doyle KM, Kennedy D, Gorman AM, et al. Unfolded proteins and endoplasmic reticulum stress in neurodegenerative disorders. J Cell Mol Med 2011;15:2025-39.

25. Li YR, King OD, Shorter J, et al. Stress granules as crucibles of ALS pathogenesis. J Cell Biol 2013;201:361-72.

26. Saxena S, Cabuy E, Caroni P. A role for motoneuron subtypeselective ER stress in disease manifestations of FALS mice. Nat Neurosci 2009;12:627-36.

27. Münch $C$, Bertolotti $A$. Self-propagation and transmission of misfolded mutant SOD1: prion or prion-like phenomenon? Cell Cycle 2011;10:1711.

28. Münch C, O'Brien J, Bertolotti A. Prion-like propagation of mutant superoxide dismutase-1 misfolding in neuronal cells. Proc Natl Acad Sci U S A 2011;108:3548-53.

29. Vaccaro A, Patten SA, Aggad D, et al. Pharmacological reduction of ER stress protects against TDP-43 neuronal toxicity in vivo. Neurobiol Dis 2013;55:64-75

30. Barbezier N, Chartier A, Bidet Y, et al. Antiprion drugs 6 -aminophenanthridine and guanabenz reduce PABPN1 toxicity and aggregation in oculopharyngeal muscular dystrophy. EMBO Mol Med 2011;3:35-49.

31. Wang L, Popko B, Tixier E, et al. Guanabenz, which enhances the unfolded protein response, ameliorates mutant SOD1-induced amyotrophic lateral sclerosis. Neurobiol Dis 2014;71:317-24.

32. Jiang $H Q$, Ren $M$, Jiang $H Z$, et al. Guanabenz delays the onset of disease symptoms, extends lifespan, improves motor performance and attenuates motor neuron loss in the SOD1 G93A mouse model of amyotrophic lateral sclerosis. Neuroscience 2014;277:132-8.

33. Das I, Krzyzosiak A, Schneider K, et al. Preventing proteostasis diseases by selective inhibition of a phosphatase regulatory subunit. Science 2015;348:239-42.

34. Vieira FG, Ping Q, Moreno AJ, et al. Guanabenz Treatment accelerates disease in a mutant SOD1 mouse Model of ALS. PLoS One 2015;10:e0135570.

35. Brooks BR, Miller RG, Swash M, et al. El Escorial revisited: revised criteria for the diagnosis of amyotrophic lateral sclerosis. Amyotroph Lateral Scler Other Motor Neuron Disord 2000;1:293-9.

36. Chiò A, Hammond ER, Mora G, et al. Development and evaluation of a clinical staging system for amyotrophic lateral sclerosis. J Neurol Neurosurg Psychiatry 2015;86:38-44.

37. Lauria G, Dalla Bella E, Antonini G, et al. Erythropoietin in amyotrophic lateral sclerosis: a multicentre, randomised, double blind, placebo controlled, phase III study. J Neurol Neurosurg Psychiatry 2015;86:879-86.

38. Tramacere I, Dalla Bella E, Chiò A, et al. The MITOS system predicts long-term survival in amyotrophic lateral sclerosis. J Neurol Neurosurg Psychiatry 2015;86:1180-5.

39. Franchignoni F, Mora G, Giordano A, et al. Evidence of multidimensionality in the ALSFRS-R Scale: a critical appraisal on its measurement properties using rasch analysis. J Neurol Neurosurg Psychiatry 2013;84:1340-5.

40. Rogers SJ. Guanabenz overdose. Ann Intern Med 1986;104:445

41. Sharp R. The Hamilton Rating Scale for Depression. Occup Med 2015;65:340. 